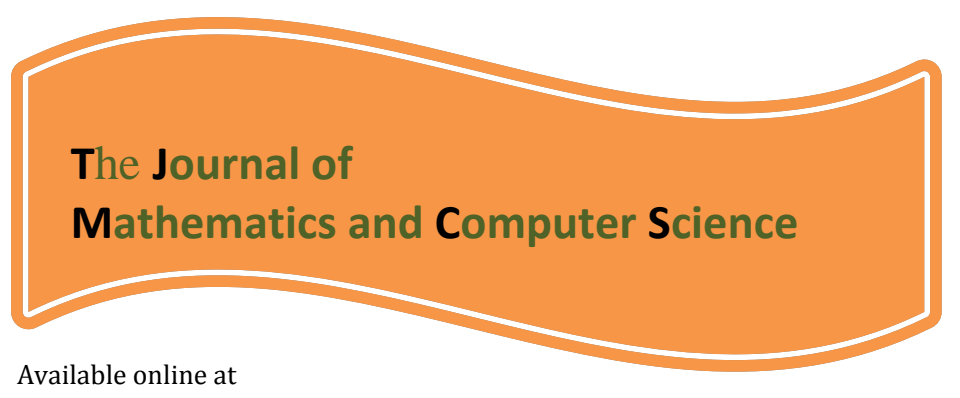

http://www.TIMCS.com

The Journal of Mathematics and Computer Science Vol .2 No.3 (2011) 448-452

\title{
Some Normal Edge-transitive Cayley Graphs on Dihedral Groups
}

\author{
A. Asghar Talebi \\ Department of mathematics \\ University of mazandaran, babolsar, Iran \\ Email: a.talebi@umz.ac.ir
}

Received: July 2010, Revised: October 2010

Online Publication: January 2011

\begin{abstract}
Let $G$ be a group and $S$ a subset of $G$ such that $1_{G} \notin S$ and $S=S^{-1}$. Let $\Gamma=\operatorname{Cay}(G, S)$ be a Cayley graph on $G$ relative to. Then $\Gamma$ is said to be normal edge-transitive, if $N_{A u t(\Gamma)}(G)$ acts transitively on edges. In this paper we determine all normal edge-transitive Cayley graphs on a dihedral Group $D_{2 n}$ of valency $n$. In addition we classify normal edge-transitive Cayley graphs $\Gamma=\operatorname{Cay}\left(D_{2 p}, S\right)$ of valency four, for a prime $p$ and give some normal edge-transitive Cayley graphs $\Gamma=\operatorname{Cay}\left(D_{2 n}, S\right)$ of valency four that $n$ is not a prime.
\end{abstract}

Keyword: Cayley graph, normal edge-transitive, Dihedral groups ,

\section{Introduction}

For a given graph $\Gamma$, we denote by $V(\Gamma), E(\Gamma)$, Aut $(\Gamma)$ the vertex set, edge set and automorphism group, respectively. Let $G$ be a group and let $S$ be a subset of $G$ such that $1_{G} \notin S$ and $S=S^{-1}$. The Cayley graph $\Gamma=\operatorname{Cay}(G, S)$ on $G$ relative to $S$ is defined by $V(\Gamma)=G, E(\Gamma)=\{\{g, s g\} \mid g \in G, s \in S\}$. The $\operatorname{graph} \Gamma=\operatorname{Cay}(G, S)$ is vertex-transitive, since $\operatorname{Aut}(\Gamma)$ contains the right regular representation $G$.Thus $G \leq \operatorname{Aut}(\operatorname{Cay}(G, S))$ and this action of 
$G$ is regular on vertices, that is, $G$ is transitive on vertices and only the identity element of $G$ fixes a vertex. A Cayley graph $\Gamma=\operatorname{Cay}(G, S)$ is said to be edge-transitive if $\operatorname{Aut}(\Gamma)$ is transitivwe on edges. In this paper graphs are finite, simple connected and undirected. It is difficult to find the full automorphism group of a graph in general, and so this makes it difficult to decide whether it is edge-trasitive. On the other hand we often have sufficien information about the group $G$ to determine $N=N_{A u t(\Gamma)}(G)$, because $N$ is the semidirect product $N=G$. Aut $(G, S)$, where $\operatorname{Aut}(G, S)=\left\{\sigma \in \operatorname{Aut}(G): S^{\sigma}=S\right\}$. Hence we focus attention on those graphs for which $N_{A u t(\Gamma)}(G)$ is transitive on edges. Such a graph is said to be normal edge-transitive. Thus it is often possible to determine whether $\operatorname{Cay}(G, S)$ is normal edge-transitive. In [5] Praeger gave an approach to analyzing normal edge-transitive Cayley graphs. Houlis in [4] determined the isomorphism types of all connected normal edge-transitive undirected Cayley graphs for $Z_{p q}$ where $p, q$ are primes, and for $G=Z_{p} \times Z_{q}, p$ a prime. In this paper we determined all normal edge-transitive Cayley graphs on a dihedral Group $D_{2 n}$ of valency $n$. In addition we classify normal edge-transitive Cayley graphs $\Gamma=\operatorname{Cay}\left(D_{2 p}, S\right)$ of valency four, for a prime $p$ and give some normal edge-transitive Cayley graphs $\Gamma=\operatorname{Cay}\left(D_{2 n}, S\right)$ of valency four such that $n$ is not a prime .

The group -and graph- theoretic notation and terminology are standard; see [2], [3], and [6] for example.

The rest of this paper is organized as follows: In the section 2 we give some preliminaries. In the section 3 we give all normal edge-transitive Cayley graphs on a dihedral group of valency $n$ and also we classify normal edge-transitive Cayley graphs $\Gamma=\operatorname{Cay}\left(D_{2 p}, S\right)$ of valency four, for a prime $p$.

\section{Preliminaries}

The following Propositions are basic for Cayley graphs.

Propositions 2.1. Let $\Gamma=\operatorname{Cay}(G, S)$ be a Cayley graph on $G$ relative to $S$. Then $\Gamma$ is connected if and only if $\mathrm{G}=\langle S\rangle$.

Let $\Gamma=\operatorname{Cay}(G, S)$ be a Cayley graph on $G$ relative to $S$, and let $A=\operatorname{Aut}(\Gamma)$. Obviously, $A \geq$ G. $\operatorname{Aut}(G, S)$. It is easy to prove the following.

\section{Propositions 2.2. [2]}

(1) $N_{A}(G)=G \cdot \operatorname{Aut}(G, S)$,

(2) $A=G$. Aut $(G, S)$ is equivalence to $G \triangleleft A$.

Propositions 2.3. [6] Let $\Gamma=\operatorname{Cay}(G, S)$ be a Cayley graph on a finite group $G$. Then $\Gamma$ is normal edge-transitive if and only if $\operatorname{Aut}(G, S)$ is either transitive on $S$ or has two orbits in $S$ which are inverses of each other. 


\section{Normal edge-transitive Cayley graphs on dihedral groups}

Throughout this section, let $D_{2 n}=\left\langle a, b: a^{2}=b^{n}=1, a^{-1} b a=b^{-1}\right\rangle$ denote the dihedral group of order $2 n$. For $n \geq 3$, any automorphism of $D_{2 n}$ can be expressed by $\sigma(r, s)$, where $b^{\sigma(r, s)}=b^{r},(n, r)=1$ and $a^{\sigma(r, s)}=a b^{s}$.

Lemma 3.1 Let $\Gamma=\operatorname{Cay}\left(D_{2 n}, S\right)$ be a normal edge-transitive Cayley graph on the dihedral group $D_{2 n}$. Then $\operatorname{Aut}\left(D_{2 n}, S\right)$ is transitive on .

Proof. Let $\Gamma=\operatorname{Cay}\left(D_{2 n}, S\right)$ be a normal edge-transitive Cayley graph for a dihedral group $D_{2 n}$. By Proposition 2.3, Aut $\left(D_{2 n}, S\right)$ is either transitive on $S$ or has two orbits in $S$ which are inverses of each other. We show that the latter case dose not arise. Let $\operatorname{Aut}\left(D_{2 n}, S\right)$ has two orbits $T$ and $T^{-1}$ such that $=T \cup T^{-1}$. Suppose that $S$ contains $a^{t}$ for some $t$, and $a^{t} \in T$. Since $\operatorname{Aut}\left(D_{2 n}, S\right)$ is transitive on $T$ and for every $\sigma \in \operatorname{Aut}\left(D_{2 n}\right),\left(a^{t}\right)^{\sigma} \in\langle a\rangle$, we have $T \subset\langle a\rangle$. Thus $S=T \cup T^{-1} \subseteq\langle a\rangle$. This is contradiction with connectivity of $\Gamma$. Hence elements of $S$ have order2, and we get $T=T^{-1}$, a contradiction.

Propositions 3.2. Let $\Gamma=\operatorname{Cay}\left(D_{2 n}, S\right)$ be a Cayley graph on the dihedral group $D_{2 n}$. Let $k$ be a positive integer with $k \geq 2$. If $S=\left\{a, a b, a b^{1+l}, a b^{1+l+l^{2}}, \ldots, a b^{1+l+\cdots+l^{k-2}}\right\}$, for an integer $l$ satisfying $1+l+\cdots+l^{k-1} \equiv 0(\bmod n)$, then $\Gamma$ is normal edge transitive.

Proof. Let $\quad \Gamma=\operatorname{Cay}\left(D_{2 n}, S\right)$ be a $\quad$ Cayley graph in which $S=\left\{a, a b, a b^{1+l}, a b^{1+l+l^{2}}, \ldots, a b^{1+l+\cdots+l^{k-2}}\right\}$, for an integer $l$ satisfying $1+l+\cdots+$ $l^{k-1} \equiv 0(\bmod n)$. Consider mapping $\alpha: D_{2 n} \rightarrow D_{2 n}$ by $b \rightarrow b^{l}, a \rightarrow a b$. Clearly, we get that $\sigma \in \operatorname{Aut}\left(D_{2 n}, S\right)$ and $\langle\alpha\rangle$ acts transitively on $S$. Then by Proposition $2.3, \Gamma=\operatorname{Cay}\left(D_{2 n}, S\right)$ is normal edge transitive.

Theorem 3.3. Let $\Gamma=\operatorname{Cay}\left(D_{2 n}, S\right)$ be a Cayley graph on the dihedral group $D_{2 n}$ of valency . Then $\Gamma$ is normal edge-transitive if and only if $S=\left\{a, a b, a b^{2}, \ldots, a b^{n-1}\right\}$.

Proof. Let $\Gamma=\operatorname{Cay}\left(D_{2 n}, S\right)$ such that $S=\left\{a, a b, a b^{2}, \ldots, a b^{n-1}\right\}$. Then $\operatorname{Aut}\left(D_{2 n}, S\right)=\operatorname{Aut}\left(D_{2 n}\right)$. We show that $\operatorname{Aut}\left(D_{2 n}\right)$ is transitive on $S$. For any $a b^{i}, 0 \leq i \leq n-1$ there exist $\sigma(r, s) \in$ $\operatorname{Aut}\left(D_{2 n}\right)$ such that $a^{\sigma(r, i)}=a b^{i}$. Hence $a^{\operatorname{Aut}\left(D_{2 n}\right)}=\mathrm{S}$ and this implies that $\operatorname{Aut}\left(D_{2 n}\right)$ is transitive on $S$. Conversely, let $\Gamma=\operatorname{Cay}\left(D_{2 n}, S\right),|S|=n$ be normal edge transitive. If $S$ contains $b^{t}$, for some $t$, then $S=\left(b^{t}\right)^{A u t\left(D_{2 n}\right)} \subseteq\langle b\rangle$ and hence $D_{2 n}=\langle S\rangle \subseteq\langle b\rangle$, a contrary. Thus $\subseteq D_{2 n}-\langle b\rangle$. Since $|S|=\left|D_{2 n}-\langle b\rangle\right|=n, S=D_{2 n}-\langle b\rangle=\left\{a, a b, \ldots ., a b^{n-1}\right\}$.

Theorem 3.4. Let $\Gamma=\operatorname{Cay}\left(D_{2 n}, S\right)$ be a Cayley graph on the dihedral group $D_{2 n}$ of valency four. If $S=\left\{a, a b, a b^{i}, a b^{1-i}\right\}$ such that $(n, 2 i-1)=1,2 i(1-i) \equiv 0(\bmod n)$ then $\Gamma$ is normal edge transitive. 
Proof. Let $\Gamma=\operatorname{Cay}\left(D_{2 n}, S\right)$ be a Cayley graph on the dihedral group $D_{2 n}$ of valency four, for $S=\left\{a, a b, a b^{2}, \ldots, a b^{n-1}\right\}$ such that $(n, 2 i-1)=1,2 i(1-i) \equiv 0(\bmod n)$.We show that $\operatorname{Aut}\left(D_{2 n}, S\right)$ is transitive on $S$. There are automorphisms $\sigma_{1}=\sigma(n-1,1), \sigma_{2}=\sigma(n-(2 i-$ $1), i)$, and $\sigma_{3}=\sigma(2 i-1,1-i)$ such that $a^{\sigma_{1}}=a b, a^{\sigma_{2}}=a b^{j}$ and $a^{\sigma_{3}}=a b^{1-i}$. Also we have $\sigma_{1}, \sigma_{2}, \sigma_{3} \in \operatorname{Aut}(G, S)$. Hence $\operatorname{Aut}\left(D_{2 n}, S\right)$ is transitive on $S$, and by Proposition $2.3 \Gamma$ is normal edge transitive.

Theorem 3.5. Let $\Gamma=\operatorname{Cay}\left(D_{2 p}, S\right)$ be a Cayley graph on the dihedral group $D_{2 p}$ of valency four, where $p$ is a prime number. Then $\Gamma$ is normal edge-transitive, if and only if $\Gamma$ is isomorphic with $\Gamma=\operatorname{Cay}\left(D_{2 p}, S\right)$ where $S=\left\{a, a b, a b^{1+l}, a b^{1+l+l^{2}}\right\}$ for an integer $l$ satisfying $1+l+l^{2}+l^{3} \equiv$ $0(\bmod n)$.

Proof. By Proposition 3.2, if $S=\left\{a, a b, a b^{1+l}, a b^{1+l+l^{2}}\right\} \quad$ for an integer $l$ satisfying $1+l+$ $l^{2}+l^{3} \equiv 0(\bmod p)$, then graph $\operatorname{Cay}\left(D_{2 p}, S\right)$ is normal edge- transitive. Conversely, let $\Gamma=\operatorname{Cay}\left(D_{2 p}, S\right)$ be a normal edge-transitive Cayley graph on the dihedral group $D_{2 p}$ of valency four. By lemma 3.1, Aut $\left(D_{2 p}, S\right)$ is transitive on $S$. Since for every automorphism $\alpha$ of $\operatorname{Aut}\left(D_{2 p}\right),\langle b\rangle^{\alpha}=\langle b\rangle, S$ is not contain $b^{t}, 0 \leq t \leq p-1$, thus we may assume that $S=$ $\left\{a, a b^{i}, a b^{j}, a b^{k}\right\}$. We have only two transitive permutation group on $S$ that are following:

$$
\begin{aligned}
& \operatorname{Aut}\left(D_{2 p}, S\right)=\left\langle\alpha=\left(a, a b^{i}, a b^{j}, a b^{k}\right)\right\rangle \\
& \operatorname{Aut}\left(D_{2 p}, S\right)=\left\langle\alpha_{1}=\left(a, a b^{i}\right)\left(a b^{j}, a b^{k}\right), \alpha_{2}=\left(a, a b^{j}\right)\left(a b^{i}, a b^{k}\right)\right\rangle
\end{aligned}
$$

In the case (I), we have $a^{\alpha}=a b^{i},\left(a b^{i}\right)^{\alpha}=a b^{j},\left(a b^{j}\right)^{\alpha}=a b^{k}$ and $\left(a b^{k}\right)^{\alpha}=a$. It follows that $\left(a \cdot a b^{i}\right)^{\alpha}=a b^{i} \cdot a b^{j},\left(a b^{i} \cdot a b^{j}\right)^{\alpha}=a b^{j} \cdot a b^{k},\left(a b^{j} \cdot a b^{k}\right)^{\alpha}=a b^{k} \cdot a$ and $\left(a b^{k} \cdot a\right)^{\alpha}=a \cdot a b^{i}$, that is $\quad\left(b^{i}\right)^{\alpha}=b^{j-i},\left(b^{j-i}\right)^{\alpha}=b^{k-j},\left(b^{k-j}\right)^{\alpha}=b^{-k}$ and $\left(b^{-k}\right)^{\alpha}=b^{i}$. Sinse $\alpha$ is a group automorphism of $\langle b\rangle$, This implies that $\left\langle b^{j}\right\rangle \subseteq\left\langle b^{i}\right\rangle,\left\langle b^{k}\right\rangle \subseteq\left\langle b^{i}\right\rangle$, and hence $\left\langle b^{i}\right\rangle \subseteq\langle b\rangle$. Thus $(i, p)=1$ and without loss of generality,we may assume that $i=1$ and $S=\left\{a, a b, a b^{j}, a b^{k}\right\}$.So we have $(a)^{\alpha}=a b,(a b)^{\alpha}=a b^{j},(a b)^{\alpha}=a b^{j},\left(a b^{j}\right)^{\alpha}=a b^{k}$ and $\left(a b^{k}\right)^{\alpha}=a$. Hence $(b)^{\alpha}=$ $(a \cdot a b)^{\alpha}=(a)^{\alpha}(a b)^{\alpha}=a b \cdot a b^{i_{2}}=b^{j-1}$ and $\quad a b^{k}=\left(a b^{j}\right)^{\alpha}=a b b^{j(j-1)}=a b^{j(j-1)+1}, a=$ $\left(a b^{k}\right)^{\alpha}=a b \cdot b^{k(j-1)}=a b^{j(j-1)^{2}+(j-1)+1}$. Let $=j-1$. Then we have $b^{k}=b^{l^{2}+l+1}$ and $1+l+l^{2}+l^{3} \equiv 0(\bmod p)$. Thus $S=\left\{a, a b, a b^{1+l}, a b^{1+l+l^{2}}\right\}$ and $1+l+l^{2}+l^{3} \equiv 0(\bmod p)$.

We show that the case (II) dos not arise. Let $\alpha_{1}=\sigma(r, i)$, which $(r, p)=1$. Then we have $\left(a b^{i}\right)^{\alpha_{1}}=a b^{i} \cdot b^{r i}=a$. Hence $b^{i+r i}=e$ and we conclude that $\mid i(1+r)$. It follows that $p=1+r$ and so $\alpha_{1}=\sigma(p-1, i)$. Furthermore, we have $a b^{k}=\left(a b^{j}\right)^{\alpha_{1}}=a b^{i} b^{j(p-1)}=a b^{i-j}$. This implies that $k \equiv i-j(\bmod p)$. Similarly, we get that $\alpha_{2}=\sigma(p-1, j)$ and $k \equiv j-i(\bmod p)$. Hence $i-j \equiv j-i(\bmod p)$. This implies that | $2(i-j)$, contrary.

In the end we give the following conjecture .

Conjecture. If $\Gamma$ be a normal edge transitive Cayley graph on dihedral group of valency four , then $\Gamma$ is isomorphism with $\operatorname{Cay}\left(D_{2 n}, S\right)$ in which either $S=\left\{a, a b, a b^{1+l}, a b^{1+l+l^{2}}\right\}$ for an integer $l$ satisfying $1+l+l^{2}+l^{3} \equiv 0(\bmod p)$ or $S=\left\{a, a b, a b^{i}, a b^{i-1}\right\}$ such that $(n, 2 i-1)=$ $1,2 i(1-i) \equiv 0(\bmod n)$. 
Proposition (3.3) shows that this conjecture holds for prime number $n$.

\section{References}

[1] N. Biggs, Algebric Graph Theory, Cambridge University Press, 1974.

[2] C. D. Godsil, On the full automorphism group of a graph, Combinatorica, 1(1981) , 243-256.

[3] C. D. Godsil, G. royle, Algebric Graph Theory, Springer-verlag, New York, 2001.

[4] P. Houlis, Quotients of normal edge-transitive Cayley graphs, M.Sc. Thesis (University of Western Australia, 1998).

[5] C. E. Praeger. Finite Normal Edge-Transitive Cayley Graphs, Bull. Austral. Math. Soc Vol. 60 (1999) 207-220.

[6] H. Wielandt, Finit Permutation Group, Academic Press, New York, 1964. 